\title{
Numerical Simulation of Shock-Dispersed Fuel Charges
}

\author{
John B. Bell, Marcus Day, Vincent Beckner, Charles Rendleman* \\ University of California Lawrence Berkeley National Laboratory, \\ Berkeley, CA USA \\ Allen L. Kuhl \\ University of California Lawrence Livermore National Laboratory, \\ Livermore, CA USA \\ P. Neuwald \\ Ernst-Mach-Institut, Fraunhofer Institut für Kurzzeitdynamik, \\ Freiburg im Breisgau, Germany
}

January 9, 2006

\section{Introduction}

For many explosives, only a fraction of the chemical energy is released in the detonation. Calorimetry data for TNT from Ornellas [7] shows that when the ambient gas is inert, there is substantially less total energy released than when the ambient gas is air. This data indicates that burning of the explosion byproducts plays a key role in the overall energetics of the system. The basic concept of shock-dispersed fuel (SDF) charges is to directly exploit this idea. More precisely, in an SDF charge a small charge is used to disperse a fuel and create a turbulent environment in which the fuel can mix with ambient air and burn.

Here, we consider a prototype SDF charge in which a $0.5 \mathrm{~g}$ PETN booster charge is used to disperse $1.0 \mathrm{~g}$ of TNT which plays the role of the fuel [6]. The hot detonation products and dispersed material are rich in fuel $\left(C_{(s)}, C O\right.$ and $\left.H_{2}\right)$, and when they mix with air and burn, they release $2,500 \mathrm{cal} / \mathrm{g}$ (in addition to the $1,100 \mathrm{cal} / \mathrm{g}$ released by the detonation) in a non-premixed turbulent combustion process [3]. The goal in this paper is to explore the dependence of the total energy release on the geometry of the calorimeter. As a baseline case, we consider a charge in air in a $6.6 \mathrm{l}$ calorimeter [6]. We first present two simulations of this baseline case corresponding to filling the calorimeter with air and filling the calorimeter with an inert gas. Comparison of these two cases illustrates the role of burning on the overall system energetics. We then discuss two series of simulations focused on exploring the dependence of the system response on problem geometry. In the first set of simulations, the aspect ratios of the calorimeter are held constant but the volume is increased. In the second set the volume is held constant but the aspect ratios are changed. In the next two sections, we briefly discuss the computational model and the numerical method used for the simulations. In the final section we present the computational results and discuss the implications of the results to the design of SDF charges.

\footnotetext{
${ }^{*}$ Corresponding author, CARendleman@lbl.gov
} 


\section{Thermodynamic model}

Developing a computational model to simulate afterburning poses something of a conundrum. One possible approach would be include a collection of chemical reactions that would capture both the chemical behavior of the detonation products as the gases expand into the calorimeter and reactions describing the oxidation of those detonation products as they mix with air. The difficulty with this approach is that even if suitable reactions were known, the resulting computation would be extremely costly. Furthermore, much of the chemical detail is relatively unimportant because the chemical time scales are very fast because of the high temperature and pressure environment.

An alternative would be to attempt to enforce some type of chemical equilibrium assumption and perform a simulation in which it is assumed that the chemical constituents are always in chemical and thermal equilibrium. However, this approach fails to accurately predict the dynamics even in the inert case in which the calorimeter is filled with nitrogen. Data from Kuhl et al. [4] shows that an equilibrium assumption does not give an accurate picture of the composition of gases resulting from a TNT charge. In essence, as the gases expand the behind the detonation, the temperature drops to the point that the reactions are effectively quenched.

Kuhl et al. [4] has developed a thermodynamic model that can accurately describe the behavior of the detonation products and provide a simplified model for the combustion process. This approach is based on a thermodynamics analysis in the Le Chatelier diagram of specific internal energy versus temperature shown in Figure 1. We note that, for these definitions, we include the chemical potential as part of the internal energy so that chemical reactions at constant volume do not change the internal energy. For this analysis we treat the gases as three components, fuel (the detonation products), air and products (the result of afterburning). The thermodynamic properties of the components $k$ (Fuel-F, Air-A, and Products-P) were computed using the Cheetah code [2] by Kuhl et al. [4]. In addition, for convenience we denote the mixture of fuel and air as the ReactantsR. When constructing the thermodynamic properties of the detonation products, we assume that the detonation products are in chemical equilibrium above $\mathrm{T}=1,800 \mathrm{~K}$ at which point the compo-

sition is frozen. This assumption produces a good approximation to the bomb calorimeter data of Ornellas [7]. The resulting data is then fit with quadratics to establish the equation of state (EOS) of the components:

$$
u_{k}\left(T_{k}\right)=a_{k} T_{k}^{2}+b_{k} T_{k}+c_{k},(k=F, A, R, P)
$$

These quadratic EOS functions (curves in Figure 1) well approximate the thermodynamic values (circles in Figure 1) and fully characterize the thermodynamic behavior of the system [4]. From these component EOS we can construct mixture thermodynamic properties based on an ideal gas approximation.

Mixture EOS:

$$
T_{m}=\left[-b_{m}+\sqrt{b_{m}^{2}-4 a_{m}\left(c_{m}-u_{m}\right)}\right] / 2 a_{m}, p_{m}=\rho_{m} R_{m} T_{m}
$$

Mixture Properties:

$$
a_{m}=\sum_{k} Y_{k} a_{k}, b_{m}=\sum_{k} Y_{k} b_{k}, c_{m}=\sum_{k} Y_{k} c_{k}, R_{m}=\sum_{k} Y_{k} R_{k}
$$

\section{Gas Dynamics}

We model the flow using the equations of inviscid, gas dynamics for the multi-component mixture $m$. Thus, the simulations implicitly incorporate a Monotone Integrated Large Eddy Simulation 


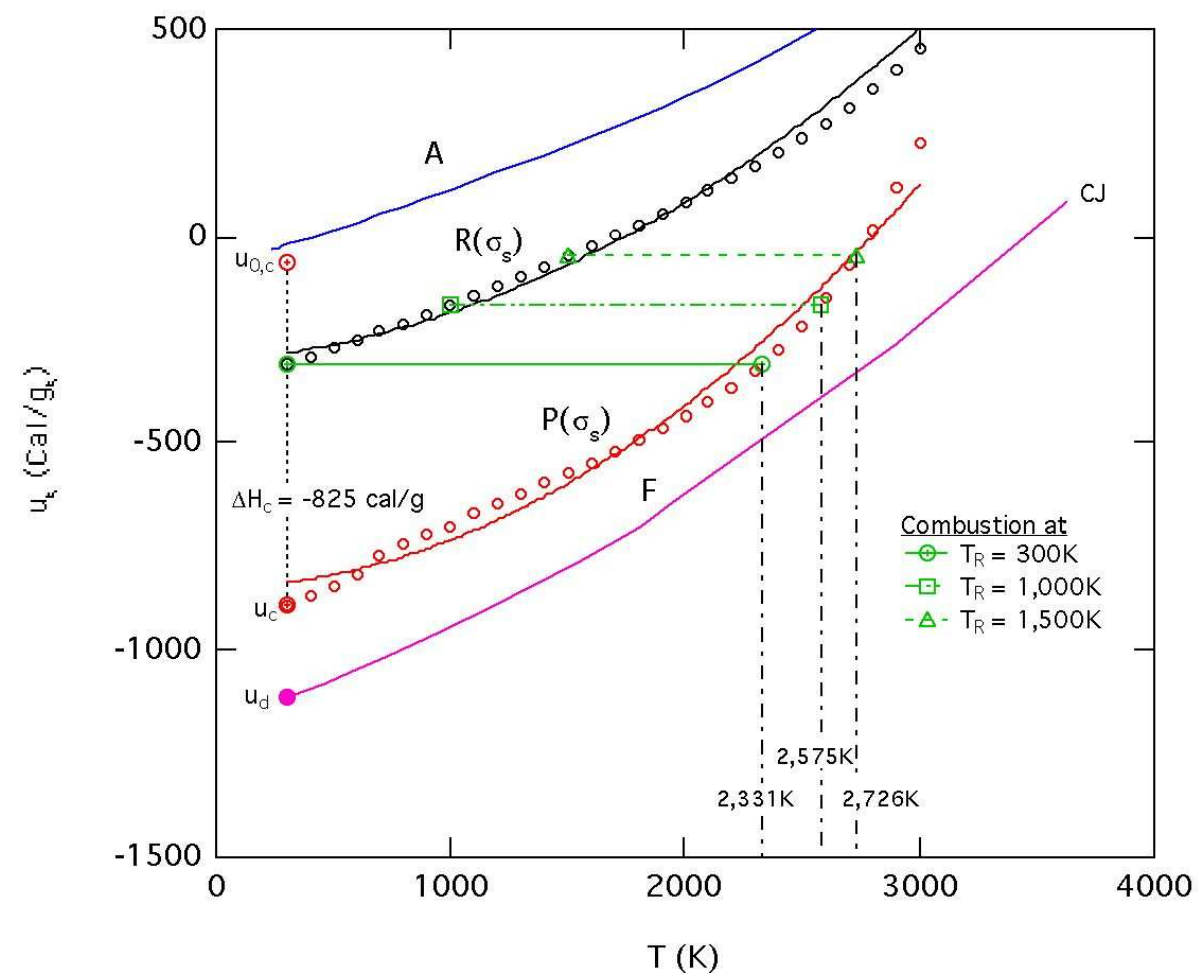

Figure 1: Locus of states in the Le Chatelier diagram for stoichiometric combustion $\left(\sigma_{s}=2.773\right)$ of the explosion products from the composite (PETN/TNT) charge in the $6.6 \mathrm{l}$ calorimeter. The green lines illustrate combustion loci starting at $300 \mathrm{~K}, 1000 \mathrm{~K}$ and $1500 \mathrm{~K}$. EOS curves are: $u_{A}(T)=1.86 \cdot 10^{-5} T^{2}+0.167 \cdot T-72.2 \mathrm{cal} / \mathrm{g}_{A}, u_{F}(T)=4.59 \cdot 10^{-5} T^{2}+0.179 \cdot T-1168 \mathrm{cal} / \mathrm{g}_{F}$, $u_{P}(T)=10.5 \cdot 10^{-5} T^{2}+0.1111 \cdot T-848 \mathrm{cal} / \mathrm{g}_{P}, u_{R}(T)=7.56 \cdot 10^{-5} T^{2}+0.042 \cdot T-302 \mathrm{cal} / \mathrm{g}_{\mathrm{R}}$, Here, the label $R\left(\sigma_{s}\right)$ and $P\left(\sigma_{s}\right)$ emphasizes that the reactants and products were computed for a stoichiometric mixture of fuel and air.

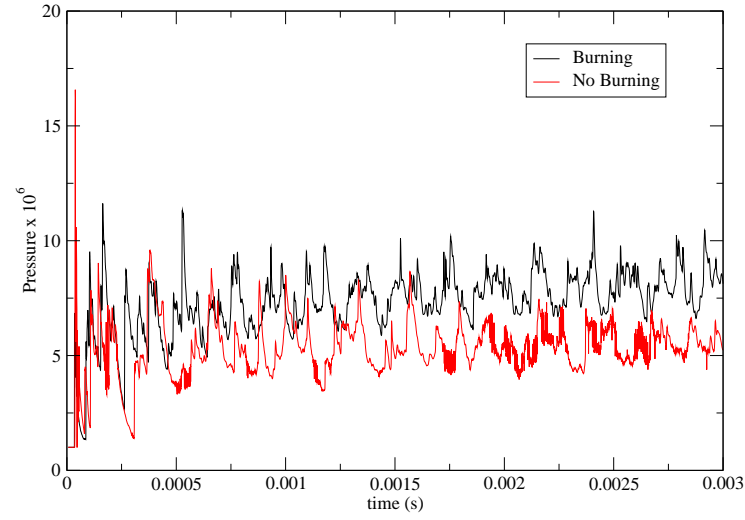

(a)

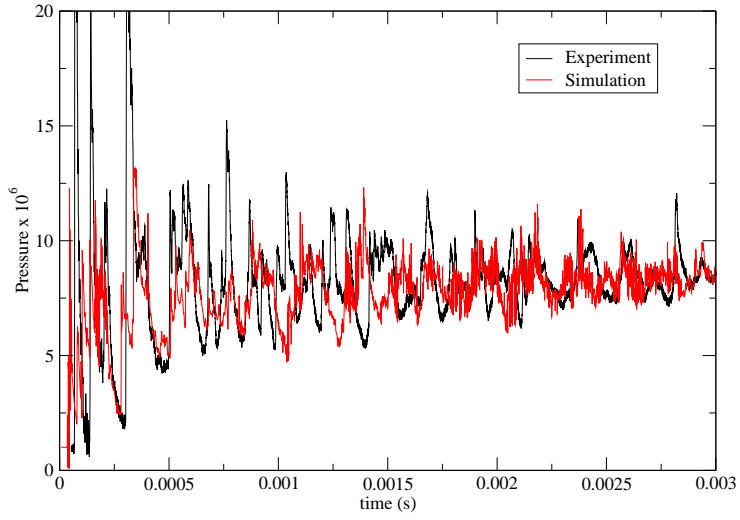

(b)

Figure 2: Pressure traces for experimental configuration A. (a) shows the pressure enhancement due to after-burning of the products of the detonation. (b) shows that the close correspondence of the result of the simulation compared to experiment 
(MILES) type representation of mixing, described by Boris et al. [1], which is a reasonable approximation to the high Reynolds and Peclet numbers associated with the post-detonation environment. More precisely, we solve

Mass:

$$
\partial_{t} \rho_{m}+\nabla \cdot\left(\rho_{m} u\right)=0
$$

Momentum:

$$
\partial_{t} \rho_{m} u+\nabla \cdot\left(\rho_{m} u u\right)=-\nabla p_{m}
$$

Energy:

$$
\partial_{t} \rho_{m}\left(u_{m}+u \cdot u / 2\right)+\nabla \cdot \rho_{m}\left(u_{m}+u \cdot u / 2\right) u=-\nabla \cdot\left(p_{m} u\right)
$$

Components:

$$
\partial_{t} Y_{k}+u \cdot \nabla Y_{k}=\alpha_{k} \dot{Y}_{s}, \quad \sum_{k} Y_{k}=1
$$

where $\rho_{m}, u_{m}$, and $p_{m}$ are the mixture density, internal energy and pressure, $u$ is the flow velocity, $Y_{k}$ are the mass fractions, $k=F, A, P$ and $\alpha_{k}=\left\{-1,-\sigma_{s},\left(1+\sigma_{s}\right)\right\}$ denotes the stoichiometric source/sink coefficients based on the stoichiometric air/fuel ratio: $\sigma_{s}=2.77$. The term $\dot{Y}_{s}$ embodies chemical kinetic effects. Here it is assumed that reactions occur on a much faster time scale than the fluid mechanics; i.e., mixtures of fuel and air instantly react to products so that mixing controls the reaction process. In addition, as noted earlier, we use a MILES approach to represent the mixing; thus the simulation represents a large-Damkhöler-number and Reynolds number limit.

The above model equations (Equations 1-4) were integrated using a high-order Godunov scheme, and Adaptive Mesh Refinement (AMR) [9] was used to follow the thin reaction zones on the computational grid. The geometry of the calorimeter is represented using an embedded boundary representation. The overall computational method is discussed in Pember et al. [8].

\section{Results}

The computations were performed in two stages. In the first stage, we simulate the initial explosion using a Jones-Wilkens-Lee (JWL) [5] equation of state to model the condensed phase behavior of the detonation products at early time. For this phase of the computation, three levels of refinement were used for an effective resolution of approximately $0.4 \mathrm{~mm}$. After the initial charge has expanded to three charge radii, the behavior is well approximated by an ideal gas. At that point, we switch to the quadratic thermodynamic model discussed above. For most of the cases we then reduce the resolution to $0.8 \mathrm{~mm}$, noting that this leads to a slight under-prediction of the overall combustion process.

For our baseline case, $\mathrm{A}$ in Table 1, the calorimeter was cylindrical with a radius of $10 \mathrm{~cm}$ and a height of $21 \mathrm{~cm}$ resulting in a total volume of $6.6 \mathrm{l}$. The computation was performed with a base mesh spacing of $3.2 \mathrm{~mm}$. In the first simulation, we include the chemical transformation corresponding to afterburning; the second does not include this effect. These two cases correspond to performing the experiment in either air or nitrogen, respectively. In Figure 2a we show pressure traces collected at a location on one end of the cylinder, $50 \mathrm{~mm}$ from the center, for the two cases. The difference in pressure illustrates the enhanced energetics resulting from afterburning. As a validation, in Figure $2 \mathrm{~b}$ we compare the simulation, including the effects of burning, done at $0.4 \mathrm{~mm}$ resolution, with the experimental data. 


\begin{tabular}{lcrrrrr}
\hline Name & Symbol & $\begin{array}{r}\text { Length } \\
(\mathrm{mm})\end{array}$ & $\begin{array}{r}\text { Diameter } \\
(\mathrm{mm})\end{array}$ & $\begin{array}{r}\text { cross- } \\
\text { section }\end{array}$ & $\begin{array}{r}\text { Volume } \\
(\mathrm{l})\end{array}$ & L/D \\
\hline $\mathrm{BBC}[6.6 / 1]$ & $\mathrm{A}$ & 210 & 200 & circular & 6.6 & 1.05 \\
$\mathrm{BBC}[21.2 / 1]$ & $\mathrm{B}$ & 300 & 300 & circular & 21.2 & 1.00 \\
$\mathrm{BBC}[40.5 / 1]$ & $\mathrm{C}$ & 379 & 369 & circular & 40.5 & 1.03 \\
$\mathrm{BBC}[6.3 / 4.6]$ & $\mathrm{D}$ & 555 & 120 & circular & 6.3 & 4.625 \\
$\mathrm{BBC}[6.3 / 12.5]$ & $\mathrm{E}$ & 1000 & 80 & square & 6.3 & 12.5 \\
\hline
\end{tabular}

Table 1: Summary of currently available barometric bomb calorimeters

Total Products

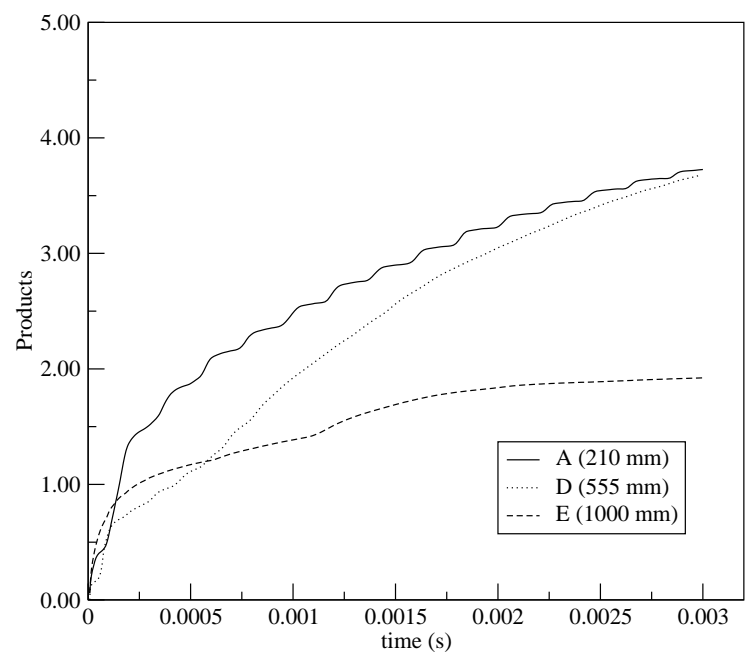

(a)
Total Products

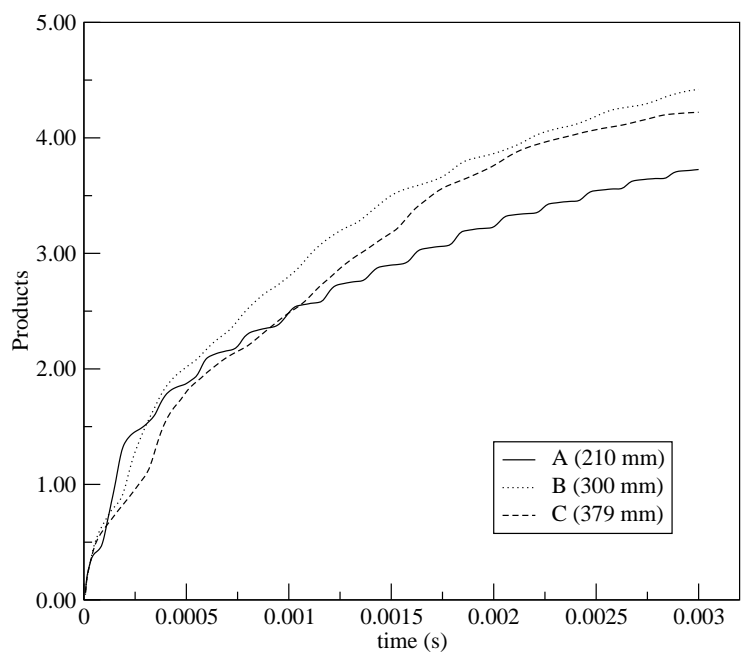

(b)

Figure 3: (a) Product histories for fixed combustor volume but changing combustor geometry, corresponding to cases A, D, and E in Table 1. (b) Product histories for fixed geometry but increasing combustor volume corresponding to cases A, B, and C in Table 1.

Two additional sets of experiments were conducted. The first set measured the effect of the volume of the calorimeter on the dynamics of the explosion. Three cylindrical calorimeters with equal shape (aspect ratio) but with increasing volume were used. The second set of experiments preserved the volume of the calorimeter but varied the shape; in one case by using a flattened cylindrical chamber, and in the second case by using a long, narrow chamber with a square crosssection. The experimental geometries, and the symbols (A-E) used to discuss them in following are described in Table 1.

Figure 3a shows the time history of integrated products for the cases in which the shape of the calorimeter is varied, but the volume is fixed at $6.6 \mathrm{l}$. It is evident that the $1000 \mathrm{~mm}$ square cross-section chamber $\mathrm{E}$ has considerably less consumption of the reactants. The reason for this is evident in the visualizations shown in Figure 4 where the fuel and oxidizer are segregated into zones separated by regions containing products.

Figure $3 \mathrm{~b}$ shows the time history of the integrated reaction products for cases in which the geometry is fixed, but the volume of the chamber is increasing. The smaller chamber, A, has the 


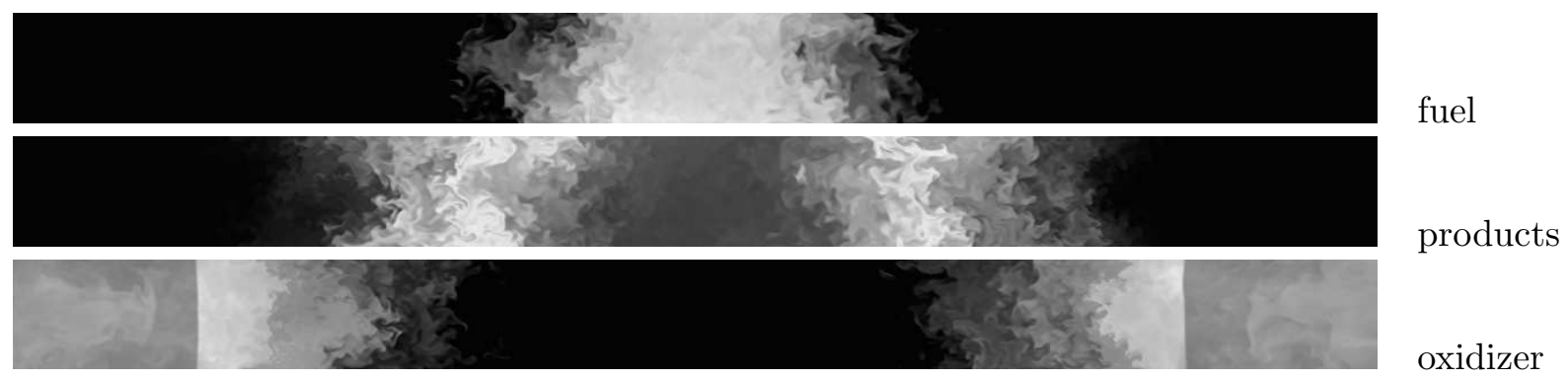

Figure 4: Fuel, products and oxidizer concentrations for case E, showing the products segregate the fuel from the oxidizer

lowest amount of integrated reaction products at late time. This is not observed in the experimental data and is mostly likely explained by the fast-reaction model for the chemical kinetics. Indeed, using the quadratic equations of state for mixture temperature given above, we can evaluate the temperature in the model calorimeter. These results, shown in Figure 5, indicate that in the larger cylinder $\mathrm{C}$ there are regions of low temperature, whereas the detonation/combustion process in the smaller chamber A has substantially raised the minimum chamber temperature. The larger cylinder $\mathrm{C}$, while cooler, has burned more reactants to products than the hotter, smaller chamber A. This suggests that the need to modify the basic model to include a threshold temperature to initiate the combustion process.

\section{Summary}

The detonation products resulting from a chemical explosion are typically not completely oxidized and will burn if mixed with oxygen at sufficiently high temperature. In this paper we have presented a computational model for the afterburning of these detonation products based on a thermodynamic model developed by Kuhl. This model captures the much of the thermodynamic and chemical behavior of the detonation products as they expand after detonation and provides a thermodynamically and chemically consistent model for their subsequent oxidation. The thermodynamic model is incorporated into a three-dimensional AMR algorithm and used to study the behavior of a composite TNT/PETN charge in a calorimeter. Additional work remains to account for temperature effects in our model, and to conduct further verification and validation through further comparisons with experimental data.

\section{References}

[1] J. Boris, F. Grinstein, E. Oran, and R. Kolbe. New insights into large eddy simulation. Fluid Dynamics Research, 10, 1992.

[2] Laurence E. Fried, P.C. Souers, W.M Howard, and P. Vitello. LLNL CHEETAH Code. http: //www-cms.llnl.gov/s-t/cheetah.html.

[3] A. L. Kuhl, R. E. Ferguson, and A. K. Oppenheim. Gasdynamics of combustion of TNT products in air. Archivum Combustionius, 19(1-4):67-89, 1999. 

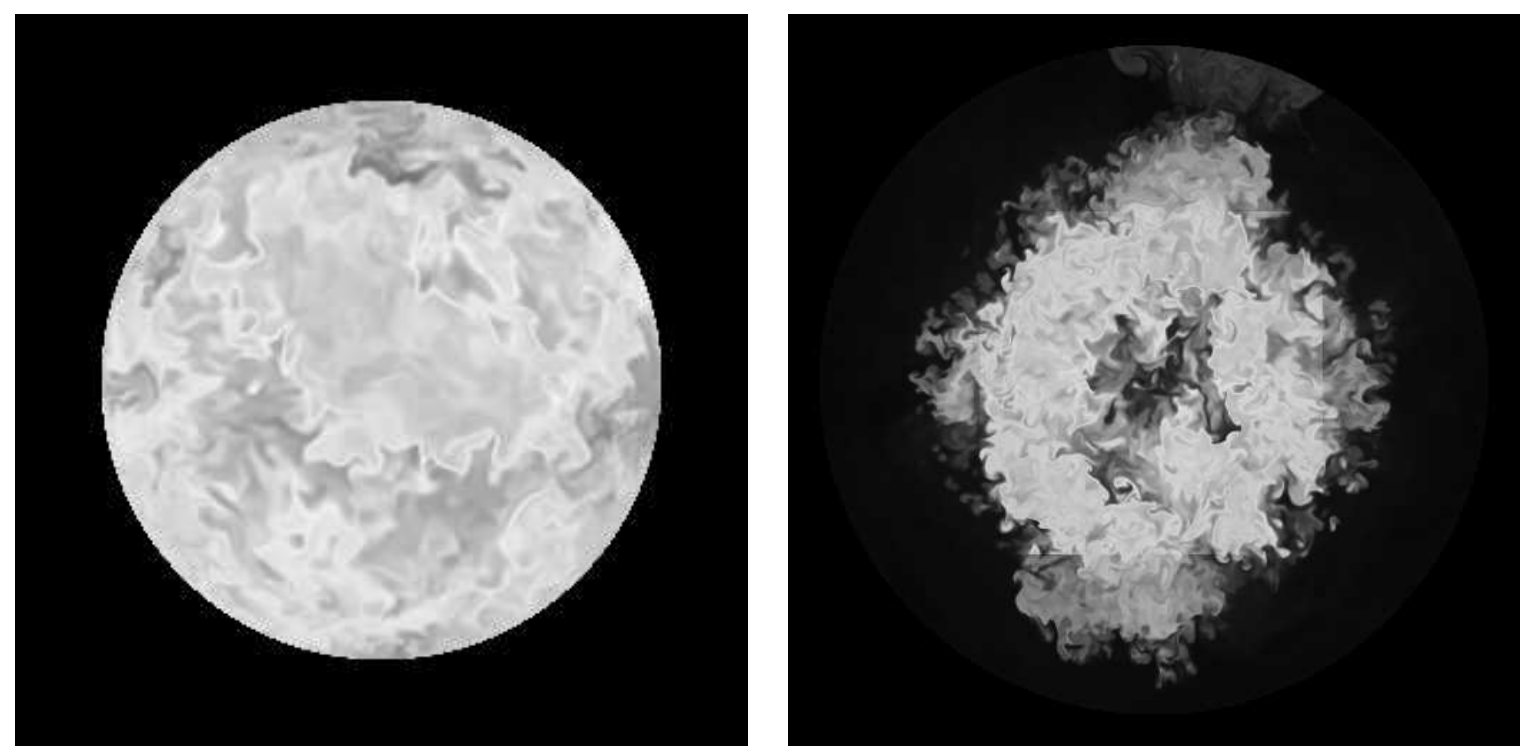

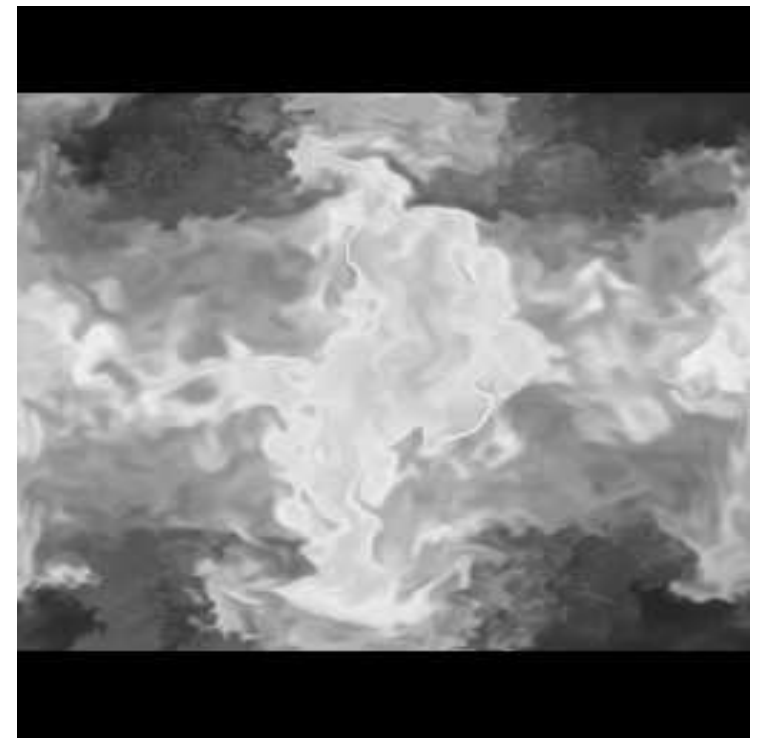

(A)

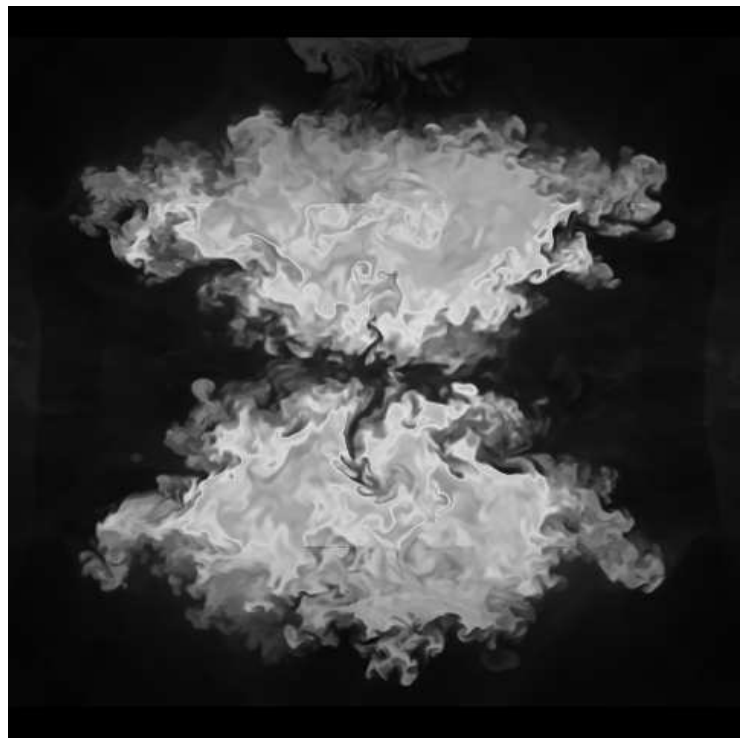

(C)

Figure 5: Model derived temperatures for models $\mathrm{A}$ and $\mathrm{C}$ from Table 1, showing significantly higher temperatures in the smaller calorimeter (brighter is higher in temperature). The first row is a slice through cross-section of the cylinder, the second row is a vertical slice through cylinder. Note: for purposes of presentation, relative volumes have not been preserved 
[4] A. L. Kuhl, M. Howard, and L. Fried. Thermodynamic model of afterburning in explosions. Energetic Materials, 34th ICT Conference, pages 74.1-74.14, 2003.

[5] E. Lee, M. Finger, and W. Collins. JWL equation of state coefficients for high explosives. Technical Report UCID-16189, Lawrence Livermore National Laboratory, Livermore, CA, 1973.

[6] P. Neuwald, H. Reichenbach, and A. L. Kuhl. Shock-dispersed fuel charges combustion in chambers and tunnels. Energetic Materials, 34th ICT Conference, pages 13.1-13.14, 2003.

[7] D. L. Ornellas. Calorimetric determination of the heat and products of detonation for explosives: October 1961 to April 1982. Technical Report UCRL-52821, Lawrence Livermore National Laboratory, Livermore, CA, 1984.

[8] R.B. Pember, J.B. Bell, P. Colella, W.Y. Crutchfield, and M.L. Welcome. An adaptive cartesian grid method for unsteady compressible flow in irregular regions. J. Comp. Phys., 120(2):278304, September 1995.

[9] Charles A. Rendleman, Vincent E. Beckner, Mike Lijewski, William Y. Crutchfield, and John B. Bell. Parallelization of structured, hierarchical adaptive mesh refinement algorithms. Computing and Visualization in Science, 3(3):147-157, 2000. 